13 Arai A, Nosaka Y, Kanda E, Yamamoto K, Miyasaka N, Miura O. Rap1 is activated by erythropoietin or interleukin-3 and is involved in regulation of beta1 integrinmediated hematopoietic cell adhesion. J Biol Chem 2001; 276: 10453-10462.

14 Mor A, Haklai R, Ben-Moshe O, Mekori YA, Kloog Y. Inhibition of contact sensitivity by farnesylthiosalicylic acid-amide, a potential Rap1 inhibitor. J Invest Dermatol 2011; 131: 2040-2048.

15 Kliche S, Worbs T, Wang X, Degen J, Patzak I, Meineke B et al. CCR7-mediated LFA- 1 functions in T cells are regulated by 2 independent ADAP/SKAP55 modules. Blood 2012; 119: 777-785. (c) (1) $\rightleftharpoons$ This work is licensed under a Creative Commons AttributionNonCommercial-NoDerivs 4.0 International License. The images or other third party material in this article are included in the article's Creative Commons license, unless indicated otherwise in the credit line; if the material is not included under the Creative Commons license, users will need to obtain permission from the license holder to reproduce the material. To view a copy of this license, visit http:// creativecommons.org/licenses/by-nc-nd/4.0/

(c) The Author(s) 2017

Supplementary Information accompanies this paper on the Leukemia website (http://www.nature.com/leu)

\title{
OPEN
}

\section{Clinical utility of gene panel-based testing for hereditary myelodysplastic syndrome/acute leukemia predisposition syndromes}

\author{
Leukemia (2017) 31, 1226-1229; doi:10.1038/leu.2017.28
}

Myelodysplastic syndrome (MDS) and acute leukemia (AL) are clinically diverse and genetically heterogeneous groups of hematological malignancies. Hereditary forms of MDS/AL were considered rare, but have been increasingly recognized in recent years. $^{1-3}$ Pathogenic variants in a single gene can predispose carriers to an increased lifetime risk of primary MDS and/or AL. Hereditary MDS/AL can occur in the context of familial MDS/AL that have MDS/AL as the principal clinical feature, or arise from inherited bone marrow failure syndromes (IBMFS), such as Fanconi anemia (FA), dyskeratosis congenita/telomerase biology disorders (TBD), Diamond-Blackfan anemia and severe congenital neutropenia. ${ }^{1,4}$ Within the past decade, nearly a dozen adult-onset familial MDS/AL syndromes have been defined. These include thrombocytopenia with associated myeloid malignancies caused by germ line mutations in RUNX1, ANKRD26 and ETV6; GATA2associated syndromes (Emberger syndrome; MonoMAC syndrome; immunodeficiency); familial MDS and acute myeloid leukemia caused by mutations in CEBPA, DDX41 and SRP72; and TBD due to mutations in TERT or TERC. ${ }^{2}$ Although the majority of patients with classic IBMFS are diagnosed in childhood, some patients have no or only subtle extra hematopoietic manifestations and may present in adulthood with MDS or AL., ${ }^{2,5}$

A few studies have shown that genetic abnormalities exist in $11-37 \%$ of families with hereditary MDS/AL. ${ }^{6-10}$ The recognition of patients with a hereditary predisposition to MDS/AL is particularly important for hematopoietic stem cell transplantation donor selection, pre-transplant planning and post-transplant care. ${ }^{11}$ The correct clinical diagnosis is also important to avoid the risk of lifethreatening toxicities with inappropriate therapy, for long-term cancer surveillance and prognosis, and for identification of at-risk or affected family members. ${ }^{5}$ Clinical guidelines for the care of MDS/AL predispositions are now emerging. ${ }^{1-3}$ To reflect the increasing recognition and clinical awareness of hereditary hematological malignancies, the World Health Organization (WHO) has included germ line predisposition to myeloid malignancies in the forthcoming WHO classification guidelines. ${ }^{12}$
However, the application of genetic testing on hereditary MDS/AL in clinical practice has never been systematically reported.

Given the phenotypic overlap of the known hereditary MDS/AL predisposition syndromes, a gene panel-based approach to genetic testing is preferred, as it offers the ability to analyze multiple genes simultaneously and cost-effectively. Our College of American Pathologists certified and Clinical Laboratory Improvement Amendments-licensed laboratory is the first to provide comprehensive clinical testing via a combination of multiple next-generation sequencing and array comparative genomic hybridization-based panel tests to evaluate genetic predisposition to MDS/AL. Multiple gene panels are available, including a familial MDS/AL panel, IBMFS panel, and panels for FA, dyskeratosis congenita/TBD, Diamond-Blackfan anemia and severe congenital neutropenia (Table 1 and Supplementary Table 1). Cultured skin fibroblasts are the preferred tissue for germ line mutation testing in patients with hematological malignancy as they provide higher quality and quantity of DNA compared to hair roots and nail clippings. The targeted nextgeneration sequencing was performed using Illumina technology (San Diego, CA, USA). The high-density exon-targeted array comparative genomic hybridization is custom designed using Agilent Technology (Santa Clara, CA, USA). The variant interpretation follows the standards and guidelines for the interpretation of sequence variants from the American College of Medical Genetics and Genomics. ${ }^{13}$

A total of 197 patients ( 110 females and 87 males) were referred to our laboratory for MDS/AL predisposition gene panel testing from October 2014 to June 2016. The patient age at the time of testing ranged from 1 to 84 years in 65 children and 132 adults. Seventy-eight patients were referred for testing for the familial MDS/AL panel, 86 for the IBMFS panel, 15 for the dyskeratosis congenita/TBD panel and 12 for multiple panel testing. In addition, a total of six patients were referred for specific testing of FA, Diamond-Blackfan anemia and severe congenital neutropenia (Table 1).

The overall molecular diagnostic rate was 19\% (37 of 197) with $15 \%$ in children and $21 \%$ in adults (Table 1). Pathogenic/likely pathogenic variants were identified in $14(18 \%)$ patients tested on the familial MDS/AL panel, $13(16 \%)$ patients tested on the IBMFS panel, 5 (33\%) patients tested on the dyskeratosis 
Table 1. Molecular diagnoses in patients tested shown by age of onset (childhood onset, 0-16 years old; adulthood onset, 17 years old and older) and all together

\begin{tabular}{|c|c|c|c|c|c|c|c|c|c|}
\hline \multirow[t]{2}{*}{ Panels } & \multicolumn{3}{|c|}{ Patients tested } & \multicolumn{3}{|c|}{ Mutation detected } & \multicolumn{3}{|c|}{ Rate of molecular diagnosis } \\
\hline & Children & Adults & Total & Children & Adults & Total & Children & Adults & Total \\
\hline IBMFS & 28 & 58 & 86 & 1 & 13 & 14 & $4 \%$ & $22 \%$ & $16 \%$ \\
\hline DC/TBD & 7 & 8 & 15 & 2 & 3 & 5 & $29 \%$ & $38 \%$ & $33 \%$ \\
\hline FA & 1 & 2 & 3 & 1 & NA & 1 & $100 \%$ & NA & $33 \%$ \\
\hline DBA & 1 & NA & 1 & 1 & NA & 1 & $100 \%$ & NA & $100 \%$ \\
\hline Total & 65 & 132 & 197 & 10 & 28 & 38 & $15 \%$ & $21 \%$ & $19 \%$ \\
\hline
\end{tabular}

Abbreviations: AML, acute myeloid leukemia; DBA, Diamond-Blackfan anemia; DC/TBD, dyskeratosis congenita/telomere biology disorders; FA, Fanconi anemia; IBMFS, inherited bone marrow failure syndrome; MDS/AL, myelodysplastic syndrome/acute leukemia; NA, not applicable; SCN, severe congenital

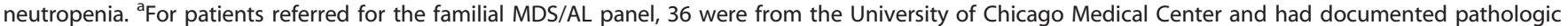

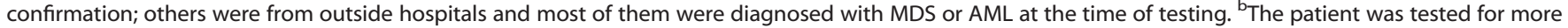
than one panel.

congenita/TBD panel, 1 (33\%) patients tested on the FA panel and $3(25 \%)$ patients tested on multiple panels. The most frequently affected genes were FANCA (four of the five cases from the IBMFS panel) and GATA2 (four of the five cases from the familial MDS/AL panel) (Table 2, Supplementary Figures 1 and 2). Unexpectedly, three of the five patients with FANCA deleterious variants were adults, and presented with aplastic anemia or acute myeloid leukemia as the major phenotype. Patient 5 was a child without typical features of FA at the time of the testing. Only Patient 2 was a child diagnosed clinically with FA. Diepoxybutane testing was performed on Patients 2 and 4, and both were abnormal. In these cases, genetic testing informed the clinical diagnosis in the absence of typical FA features. Our result is also consistent with previous reports that GATA2 is one of the more commonly mutated genes in MDS/AL predisposition syndromes. ${ }^{8,14}$ Four of the five GATA2 deleterious variants were novel, and four of them were located in the ZF2 domain, further emphasizing this domain as a mutational hot spot (Table 2 and Supplementary Figure 2). TERT, DDX41 and RUNX1 were the next frequently mutated genes (Table 2 and Supplementary Figure 2). Overall, 21 novel pathogenic/likely pathogenic variants have been identified (Table 2).

Gene panel testing can aid in the clinical diagnosis of hereditary MDS/AL, particularly in the presence of phenotypic overlap and genetic heterogeneity. For instance, Patient 4, a 19-year-old male with a history of longstanding unexplained thrombocytopenia and learning disability, developed acute myeloid leukemia and was suspected to carry a pathogenic variant in RUNX1, ETV6 or ANKRD26 (Table 2). However, testing detected two pathogenic variants in FANCA, c.2398G > T (p.Glu800*) and c.2601+1G > T (p.?) (Table 2), supporting a diagnosis of FA. Subsequent diepoxybutane testing confirmed the diagnosis of FA and prompted changes in the medical management to avoid substantial morbidity due to intensive chemotherapy. ${ }^{15}$

Our testing has also demonstrated that the identification of pathogenic variants predisposing to MDS/AL has a significant impact on the choice of pre-transplant conditioning and selection of sibling donors. A pathogenic variant in GATA2 was identified in Patient 8 who was being evaluated for hematopoietic stem cell transplantation at the time of testing, with a sister as a potential donor. The molecular diagnosis in this patient urged follow-up testing to be performed on the sister to prevent potentially devastating consequences associated with the use of a donor who carries the same pathogenic GATA2 variant.

A total of 106 variants of uncertain significance were identified in $72(37 \%)$ patients (Supplementary Figure 3). As the majority of the genes in these panels are relatively novel, less is known about unique variants seen for the first time. Population genetic data, segregation studies in family members and functional studies may help clarify the nature of some of these variants and reduce the yield of variants of uncertain significance on similar panels. Among the variants of uncertain significance, two were predicted to affect RNA splicing by in silico prediction tools (Alamut Visual, Rouen, France), and RNA splicing assays were performed to clarify the nature of these variants. The first, a novel heterozygous FANCA variant, c.826+5_826+9del (p.?), in Patient 4, was predicted to affect the canonical splice donor site of exon 9 (Table 2, Supplementary Table 2, Supplementary Figure 4A). RNA splicing assay revealed the presence of an aberrant isoform with a deletion of exon 9 that resulted in a premature stop codon in exon 10 (Supplementary Figures 4B-D). Further review of the nextgeneration sequencing data revealed an additional large heterozygous deletion of exons 21-28 in FANCA in this patient, which was confirmed by array comparative genomic hybridization (Supplementary Figure $4 \mathrm{E}$ ) and determined to be in trans with c.826+5_826+9del. The second, a novel GATA2 variant, c.857C > T (p.Ala286Val), in Patient 6, was predicted to generate a cryptic splice donor site (Table 2 and Supplementary Table 2). We demonstrated an aberrant isoform with a deletion of $16 \mathrm{bp}$ in exon 3 resulting in a premature stop codon in exon 4 of GATA2 by RNA splicing analysis of skin fibroblasts with or without cycloheximide treatment (a nonsense-mediated messenger RNA decay inhibitor; Supplementary Figures $5 \mathrm{~A}$ and $\mathrm{B}$ ). The results provided sufficient evidence that the $c .857 C>T$ is a likely pathogenic variant.

Eighty-seven (44\%) patients had negative testing results. Genetic abnormalities in a considerable proportion of patients with a history of MDS/AL predisposition therefore remain uncharacterized, suggesting that additional germ line genetic aberrations exist and remain to be identified. Research studies and periodic follow-up will help establish the genetic basis of the disorders in these patients. Clinical genetic testing also needs to be continually updated with the rapidly growing recognition of additional MDS/AL risk genes and syndromes.

In conclusion, our study demonstrates the utility of genetic testing for hereditary MDS/AL predisposition syndromes. This study has provided a better understanding of the genetic etiology of hereditary MDS/AL predisposition syndromes and broadened the gene mutation spectrum. RNA splicing analysis played an important role in clarifying variant pathogenicity. The cost of these panels is similar to gene panel testing for other genetic disorders. Our gene panel-based testing for the diagnosis of hereditary 


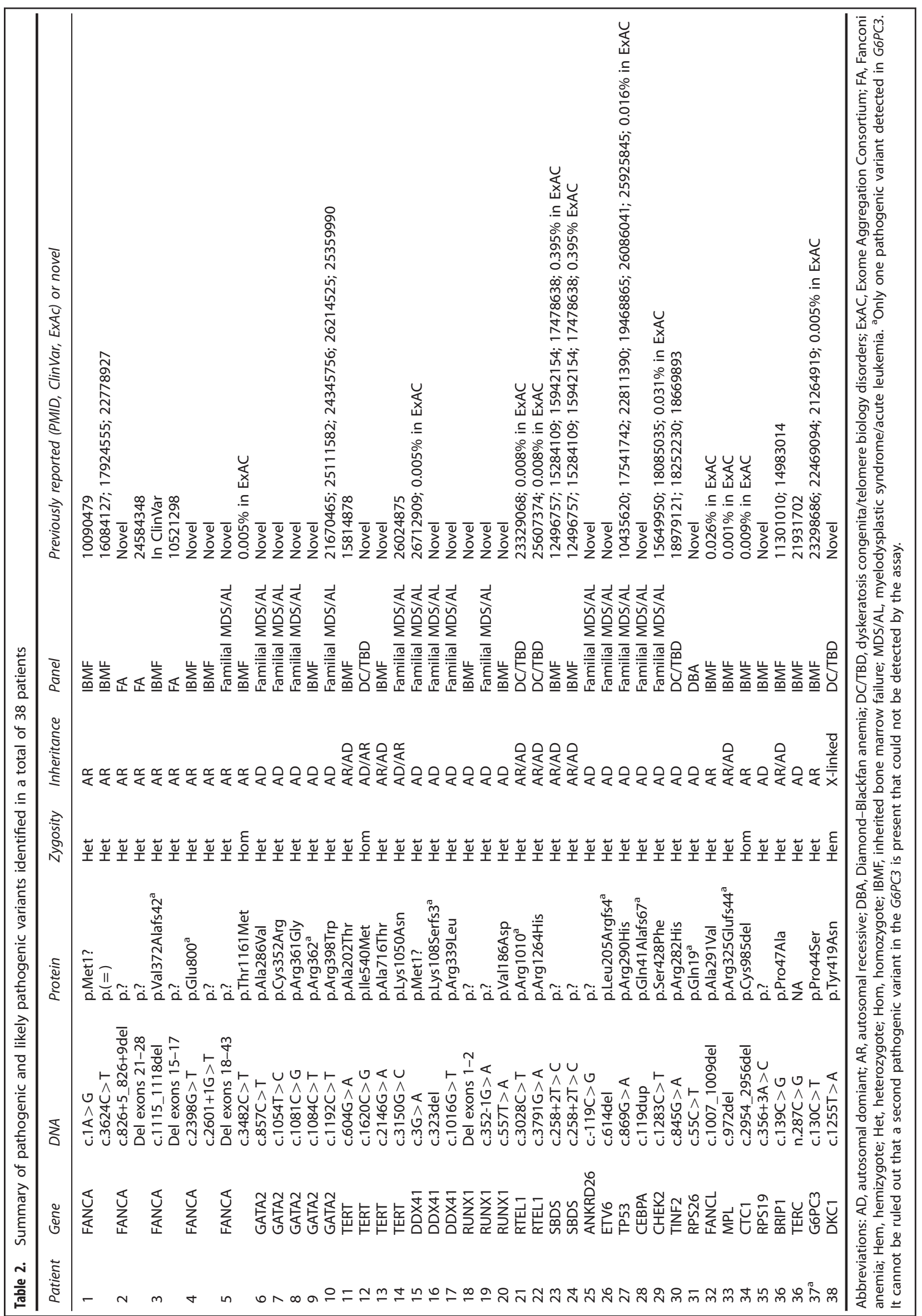


MDS/AL syndromes is being integrated into clinical hematological malignancy evaluation and the clinical decision-making for personalized treatment considerations.

\section{CONFLICT OF INTEREST}

The authors declare no conflict of interest.

\section{ACKNOWLEDGEMENTS}

We thank Darrel Waggoner for helpful discussion, Frances Kobiernicki, David Fischer, Kirsten Donato, Ying Ying Hu, Priscilla Sodadasi, Latrice Wysinger, Alexander Gary and Lan Ma for sample processing. This work was supported in part by the Institutional Research Grant from the American Cancer Society (ZL).

\section{AUTHOR CONTRIBUTIONS}

LG, GA-A and KA performed the experiments. ZL, LG, AKJ, VN, JEC, LAG, DT, NSY, $C F$, DdG and SD analyzed and interpreted the data. ZL and LG wrote the paper. ZL designed and coordinated the research study.

L Guidugli ${ }^{1}$, AK Johnson ${ }^{1}$, G Alkorta-Aranburu ${ }^{1}$, V Nelakuditi ${ }^{1}$, $\mathrm{K} \mathrm{Arndt}^{1}$, JE Churpek ${ }^{2}$, LA Godley ${ }^{2}$, D Townsley ${ }^{3}$, NS Young ${ }^{3}$, C Fitzpatrick ${ }^{4}$, D del Gaudio ${ }^{1}$, S Das ${ }^{1}$ and $Z \mathrm{Li}^{1}$

${ }^{1}$ Department of Human Genetics, The University of Chicago, Chicago, IL, USA;

${ }^{2}$ Department of Medicine, Comprehensive Cancer Center and Center for Clinical Cancer Genetics, The University of Chicago, Chicago, IL,

USA;

${ }^{3}$ Hematology Branch, Cell Biology Section, National Institutes of Health, National Heart, Lung, and Blood Institute, Bethesda, MD, USA

and

${ }^{4}$ Department of Pathology, The University of Chicago, Chicago, IL, USA

E-mail: zli7@bsd.uchicago.edu
4 Owen C, Barnett M, Fitzgibbon J. Familial myelodysplasia and acute myeloid leukaemia--a review. Br J Haematol 2008; 140: 123-132.

5 Babushok DV, Bessler M. Genetic predisposition syndromes: when should they be considered in the work-up of MDS? Best Pract Res Clin Haematol 2015; 28: 55-68.

6 Holme H, Hossain U, Kirwan M, Walne A, Vulliamy T, Dokal I. Marked genetic heterogeneity in familial myelodysplasia/acute myeloid leukaemia. $\mathrm{Br} J \mathrm{Haematol}$ 2012; 158: 242-248.

7 DiNardo CD, Bannon SA, Routbort M, Franklin A, Mork M, Armanios M et al. Evaluation of patients and families with concern for predispositions to hematologic malignancies within the hereditary hematologic malignancy clinic (HHMC). Clin Lymphoma Myeloma Leuk 2016; 16: 417-428.e2.

8 Churpek JE, Pyrtel K, Kanchi KL, Shao J, Koboldt D, Miller CA et al. Genomic analysis of germ line and somatic variants in familial myelodysplasia/acute myeloid leukemia. Blood 2015; 126: 2484-2490.

9 Keel SB, Scott A, Sanchez-Bonilla M, Ho PA, Gulsuner S, Pritchard CC et al. Genetic features of myelodysplastic syndrome and aplastic anemia in pediatric and young adult patients. Haematologica 2016; 101: 1343-1350.

10 Zhang MY, Keel SB, Walsh T, Lee MK, Gulsuner S, Watts AC et al. Genomic analysis of bone marrow failure and myelodysplastic syndromes reveals phenotypic and diagnostic complexity. Haematologica 2015; 100: 42-48.

11 Churpek JE, Artz A, Bishop M, Liu H, Godley LA. Correspondence regarding the consensus statement from the Worldwide Network for Blood and Marrow Transplantation Standing Committee on donor issues. Biol Blood Marrow Transplant 2016; 22: 183-184.

12 Arber DA, Orazi A, Hasserjian R, Thiele J, Borowitz MJ, Le Beau MM et al. The 2016 revision to the World Health Organization classification of myeloid neoplasms and acute leukemia. Blood 2016; 127: 2391-2405.

13 Richards S, Aziz N, Bale S, Bick D, Das S, Gastier-Foster J et al. Standards and guidelines for the interpretation of sequence variants: a joint consensus recommendation of the American College of Medical Genetics and Genomics and the Association for Molecular Pathology. Genet Med 2015; 17: 405-424.

14 Pasquet M, Bellanne-Chantelot C, Tavitian S, Prade N, Beaupain B, Larochelle O et al. High frequency of GATA2 mutations in patients with mild chronic neutropenia evolving to MonoMac syndrome, myelodysplasia, and acute myeloid leukemia. Blood 2013; 121: 822-829.

15 Talbot A, Peffault de Latour R, Raffoux E, Buchbinder N, Vigouroux S, Milpied N et al. Sequential treatment for allogeneic hematopoietic stem cell transplantation in Fanconi anemia with acute myeloid leukemia. Haematologica 2014; 99: e199-e200.

\section{REFERENCES}

1 Churpek JE, Lorenz R, Nedumgottil S, Onel K, Olopade OI, Sorrell A et al. Proposal for the clinical detection and management of patients and their family members with familial myelodysplastic syndrome/acute leukemia predisposition syndromes. Leuk Lymphoma 2013; 54: 28-35.

2 Bannon SA, DiNardo CD. Hereditary predispositions to myelodysplastic syndrome. Int J Mol Sci 2016; 17: 1-11.

3 Babushok DV, Bessler M, Olson TS. Genetic predisposition to myelodysplastic syndrome and acute myeloid leukemia in children and young adults. Leuk Lymphoma 2016; 57: 520-536.

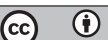

This work is licensed under a Creative Commons Attribution 4.0 International License. The images or other third party material in this article are included in the article's Creative Commons license, unless indicated otherwise in the credit line; if the material is not included under the Creative Commons license, users will need to obtain permission from the license holder to reproduce the material. To view a copy of this license, visit http://creativecommons.org/licenses/ by/4.0/

(c) The Author(s) 2017

Supplementary Information accompanies this paper on the Leukemia website (http://www.nature.com/leu)

\section{Deletion of Ptpn1 induces myeloproliferative neoplasm}

Leukemia (2017) 31, 1229-1234; doi:10.1038/leu.2017.31

Deletion of chromosome $20 \mathrm{q}(\mathrm{del}(20 \mathrm{q}))$ is a common chromosomal abnormality associated with myeloid neoplasms including myeloproliferative neoplasms (MPNs), myelodysplastic syndrome, myelodysplastic syndrome/MPN overlap disorders and acute myeloid leukemia. ${ }^{1,2}$ The del $(20 \mathrm{q})$ lesion is present in patients with myelofibrosis (MF) at a high frequency (23\%) and is thus considered to be one of the most frequent cytogenetic abnormalities in MF. ${ }^{3}$ However, the identity of the target tumor suppressor gene(s) within $20 \mathrm{q}$ involved in the pathogenesis of MF and other myeloid neoplasms remains elusive.

The PTPN1 gene encoding protein tyrosine phosphatase nonreceptor type 1 (PTPN1; also known as PTP1B) is located on human chromosome 20q13.1-q13.2. Both oncogenic and tumor suppressor functions for PTPN1 have been suggested. PTPN1 is overexpressed in breast cancer and deletion of Ptpn1 inhibits ErbB2- 